\title{
Effect of Melissa officinalis $L$. leaf extract on lipid accumulation by modulating specific adipogenic gene transcription factors in 3T3-L1 adipocytes
}

\author{
Hyun Jeong Lee ${ }^{1} \cdot$ Jonghak Lim $^{2} \cdot J_{u n o h ~ P e a k}{ }^{3} \cdot$ Mun-sang $\mathrm{Ki}^{4} \cdot$ Sang-bong Lee $^{5}$ - Gayong Choe ${ }^{6}$. \\ Jaeyun Jung ${ }^{7} \cdot$ Hansang Jung $^{8} \cdot$ Suwon Jeon $^{9} \cdot$ Tae-Sik Park $^{9} \cdot$ Soon-Mi Shim $^{1}$ (D)
}

Received: 3 June 2020 / Accepted: 16 June 2020 / Published Online: 30 June 2020

(C) The Korean Society for Applied Biological Chemistry 2020

\begin{abstract}
The objective of this study was to investigate the effects of a hypodermic injectable solution comprised of an LPM LB meso solution containing Melissa officinalis L. leaf extract (LPM) on the lipogenesis in the 3T3-L1 cells line. The lipid accumulation measured by oil red o staining in the 3T3-L1 adipocytes treated with LPM, which was reduced in a dose dependent manner and showed 91.7 to $62.9 \%$ compared to control
\end{abstract}

Soon-Mi Shim $(\bowtie)$

E-mail: soonmishim@sejong.ac.kr

${ }^{1}$ Department of Food Science and Technology, Sejong University, 98 Gunja-dong, Gwangjin-gu, Seoul 143-747, Republic of Korea

${ }^{2}$ Kairos Clinic, \# 205 Shinan Metro Khan 10 Teheran-ro 88-gil, Gangnamgu, Seoul 06179, Republic of Korea

${ }^{3}$ The Jun Skin Clinic, \#2 Chung-heung class, Kwang-gyuo Hosu-gongwon Ro 277, Youngtong-gu, Suwon, Gyeonggi-do 16517, Republic of Korea

${ }^{4} \mathrm{NB}$ Clinic, 76, Gojan-ro, Danwon-gu, Ansan-si, Gyeonggi-do, Republic of Korea

${ }^{5}$ Wonjin Skin Care Clinic, 419, Gangnam-daero, Seocho-gu, Seoul 15360, Republic of Korea

${ }^{6}$ Amiskin Clinic, 66-1, Namdaemun-ro 1-gil, Jung-gu, Seoul 04535, Republic of Korea

${ }^{7}$ Mielle Dermatology \& Plastic Surgery Network Clinic Clinic, 48, Seomyeon-ro, Busanjin-gu, Busan 47286, Republic of Korea

${ }^{8}$ Department of Physical Education, Kangnam University, Yongin-si, Gyeonggi-do 16979, Republic of Korea

${ }^{9}$ Department of Life Science, Gachon University, Sungnam, Gyeonggi-do 13120, Republic of Korea

This is an Open Access article distributed under the terms of the Creative Commons Attribution Non-Commercial License (http://creativecommons. org/licenses/by-nc/3.0/) which permits unrestricted non-commercial use, distribution, and reproduction in any medium, provided the original work is properly cited. group. Its effectiveness with a $50 \%$ solution was significantly higher than the hydroxycitric acid (positive control) treatment without showing cell cytotoxicity. In a quantitative real-time PCR, it was demonstrated that the LPM treatment appeared to upregulate the mRNA expression of the adipogenesis-related genes, which included the peroxisome proliferator-activated receptor gamma (50\% concentration) while down-regulating the CCAATenhancer binding protein alpha (50\% concentration) and the sterol regulatory element-binding protein $1 \mathrm{c}(10,25$, and $50 \%$ concentrations). The results from the current study suggest that the LPM could be useful biomaterials that can inhibit obesity in the 3T3-L1 cells, which could possibly be by regulating the specific adipogenic gene transcription factors.

Keywords Adipocytes - Adipogenic gene $\cdot$ Lipid accumulation - Melissa officinalis L. leaf · 3T3-L1 cell

\section{Introduction}

The formation of the adipocytes through adipogenesis, differentiation of the pre-adipocytes to the mature adipocytes from the cell expansion leads to the enhanced lipid accumulation, and it causes obesity [1]. Obesity causes complicated health problems that include dyslipidemia, carcinogenesis, cardiovascular disease, and type-2 diabetes [2]. Common strategies to reduce the incidences of obesity are diet changes, drug treatments, physical activity, and surgery [3]. The United States Food and Drug Administration has approved drugs for obesity treatments, such as orlistat and lorcaserin to combat obesity, but the consumers and the pharmaceutical industry are looking for an alternative solution due to the side effects and the low success rate [3,4]. Natural bioactive substances extracted from plants, which are known to provide 
various biological benefits in numerous body systems, have been suggested to prevent obesity [5]. In particular, natural compounds derived from food are considered safe and acceptable for patients compared to synthetic therapeutics [6]. It is essential to establish an exact and a non-toxic dose of natural compounds within a cell system in order to confirm the quality and the safety of a therapeutic natural product [7].

Melissa officinalis L. (Labiatae; lemon balm) is a medicinal plant that has been widely used as an ethno medical treatment for cardiovascular disease, inflammation, neurotoxicity, and depression $[7,8]$. Its biological activities, particularly the antioxidant activity, is attributed to the phenolic acids, such as rosmarinic acid, gallic acid, and caffeic acid [8]. In addition, it was reported that a diet supplemented with lemon balm extract that mainly contained caffeic acid and rosmarinic acid reduced the adipogenesis in 3T3L1 and inhibited the adipocyte hypertrophy in high fat dietinduced obese C57BL/6J mice [9]. Regarding injectable biologics to stimulate lipolysis, research interests regarding the use of natural compounds have shown to prove the clinical efficacy with an oral intake are growing due to safety [10]. Even though lemon balm has been shown to provide lipolysis activity, the effect of a hypodermic injectable solution that consisted of lemon balm leaf extracts as whole natural products on lipogenesis has not been studied.

The differentiation process of adipocytes is modulated through the inter-connected regulation of transcription factors that include CCAAT-enhancer binding protein (CEBP), a peroxisome proliferatoractivated receptor (PPAR), and sterol regulatory element-binding protein (SREBP) [1,4]. Among the adipogenic transcription factors, the CCAAT-enhancer binding protein alpha $(\mathrm{CEBP} \alpha)$ is involved in the late stages of the adipogenesis process and regulates the expression of the target genes that lead to the adipocyte development [11]. The peroxisome proliferator-activated receptors (PPARs) ligands are one of the main factors for the change of the obesity-associated comorbidities and for the energy balance [12]. Particularly, the peroxisome proliferator-activated receptor gamma (PPAR $\gamma$ ) is known to play a role in the multiple metabolic action and the cellular action, which include the regulation of lipid metabolism, anti-inflammatory properties, and the suppression of oxidative stress [13,14]. Activation of the PPAR $\gamma$ leads to stimulate the development and the differentiation of the fresh adipocytes in the 3 T3 cells $[15,16]$. The SREBP is another transcription factor involved in the cell adipogenesis, the lipid homeostasis, and the adipocyte differentiation [17].

Thus, the purpose of the current study was to evaluate the effect of the LPM LB meso solution containing Melissa officinalis L. leaf extract (LPM) on the cell cytotoxicity, the lipid accumulation, and the adipocytes gene modulation by various transcription factors, which included CEBP $\alpha$, CCAAT-enhancer binding protein beta (CEBP $\beta)$, PPAR $\gamma$, and SREBP1, by using the 3T3-preadipocyte cell model system.

\section{Materials and Methods}

\section{Sample preparations}

A hypodermic injectable LPM LB meso solution containing Melissa officinalis $L$. leaf extract (LPM) was obtained from SKY BIO. In detail, the LPM was mixed with about $0.02 \%$ mellissa officinalis leaf extract, aqua, sodium chloride, propylene glycol, methylpropanedial, tyrosine, aesculus hippocastanum (horsechestnut) seed extract, juglans regia (walnut) seed extract, focus vesiculosus extract, methysilanol mannuronate, sorbic acid, adenosine phosphate, and disodium adenosine triphosphate.

\section{T3-L1 cell culture and adipocytes}

The 3T3-L1 cells obtained from the American Type Culture Collection (ATCC, Manassas, VA) were maintained containing Dulbecco's modified Eagle's medium (DMEM, Biotechnics Research, Inc., Lake Forest, CA, USA) supplemented with a $10 \%$ bovine calf serum (Gibco, Thermo Fisher Scientific, Waltham, MA, USA) and a $1 \%$ penicillin/streptomycin solution (Biotechnics Research, Inc., Lake Forest, CA, USA) and incubated to culture conditions at $37{ }^{\circ} \mathrm{C}$ in $5 \% \mathrm{CO}_{2} .10 \mu \mathrm{g} / \mathrm{mL}$ of insulin in a DMEM containing a $10 \%$ fetal bovine serum (FBS), $0.5 \mathrm{mM}$ of isobutylmethylxanthine, and $0.5 \mu \mathrm{M}$ of dexamethasone was treated into the 3T3-L1 preadipocytes for 2 days to induce adipocyte differentiation. Then, the cells were maintained in a $10 \% \mathrm{FBS} / \mathrm{DMEM}$ medium with $10 \mu \mathrm{g} / \mathrm{mL}$ of insulin for another 2 days (D4), and they were then cultured for an additional 4 days (D8). On D9, the cells that had more than a $90 \%$ differentiation with the lipid content were quantified by measuring the absorbance at $500 \mathrm{~nm}$ using a microplate reader (Thermo Scientific, San Jose, CA, USA).

\section{Measurement of Cell Viability}

3-(4-5-dimethylthiazol-2-yl)-2.5-dyphenyltetrazolium bromide (MTT) assay to measure the metabolic activity and against the cell cytotoxicity was conducted in the 3T3-L1 adipocytes. A 3T3-L1 cell $\left(5 \times 10^{3} /\right.$ well) was seeded in 96 well plates (SPL life Science, Pocheon, Republic of Korea), and it was then treated with various doses (1, $5,10,25,50$, and $100 \%$ ) of the products. It was stained with a 200 $\mu \mathrm{L}$ MTT solution and then incubated for $4 \mathrm{~h}$ at $37{ }^{\circ} \mathrm{C}$. After the incubation, $100 \mu \mathrm{L}$ of dimethyl sulfoxide was added to solubilize the resultant formazan crystals. The values of the cell viability were measured at $570 \mathrm{~nm}$ using a microplate reader (Thermo Scientific):

\footnotetext{
Cell viability (\%)

$=$ Optical density (Average of sample - Average of blank) $\times 100$

Optical density (Average of control - Average of blank)
} 
Table 1 Primers for sequences of transcription factors including enhancer-binding protein (CEBP), peroxisome proliferator-activated receptor (PPAR), and sterol regulatory element-binding protein (SREBP)

\begin{tabular}{ccl}
\hline \hline Target Gene & Direction & \multicolumn{1}{c}{ Sequence (5'-3') } \\
\hline \multirow{2}{*}{$\beta$-Actin } & $\mathrm{F}$ & AGA GCT ACG AGC TGC CTG AC \\
& $\mathrm{R}$ & AGC ACT GTG TTG GCG TAC AG \\
\hline \multirow{2}{*}{ CEBP $\alpha$} & $\mathrm{F}$ & GAACAGCAACGAGTACCGGGTA \\
& $\mathrm{R}$ & GCCATGGCCTTGACCAAGGAG \\
\hline \multirow{2}{*}{ CEBP $\beta$} & $\mathrm{F}$ & GGAGACGCAGCACAAGGT \\
& $\mathrm{R}$ & AGCTGCTTGAACAAGTTCCG \\
SREBP1c & $\mathrm{F}$ & ATGGATTGCACATTTGAAGACATGCTC \\
& $\mathrm{R}$ & CCTGGGCTGCTGGGGCCT \\
\hline \multirow{2}{*}{ PPAR $\gamma$} & $\mathrm{F}$ & CCAGAGCATGGTGCCTTCGCT \\
& $\mathrm{R}$ & CAGCAACCATTGGGTCAGCTC
\end{tabular}

\section{Lipid accumulation by oil red o staining}

The degree of adipocyte hypertrophy was measured as the lipid accumulation by the oil red $\mathrm{O}$ staining in the $3 \mathrm{~T} 3$ cells. PBS was then fixed in a $3.7 \%(\mathrm{w} / \mathrm{v})$ formaldehyde solution that was used for washing the $3 \mathrm{~T} 3$ cells, and they were then washed again in distilled water and a $60 \%$ isopropanol solution. The cells treated with the oil red o solution were kept in an incubator for 1 hour. Then, the stained cells were washed with distilled water several times before they were photographed. The images were analyzed using an optical microscope (OLYMPUS model CKX41SF, Tokyo, Japan) at a $200 \times$ magnification and then spectrophotometric quantification was conducted at $500 \mathrm{~nm}$.

\section{mRNA preparation and Quantitative Real-time PCR}

Total RNA was extracted from the cells using an easy-spin total RNA extraction kit (Intron Biotechnology, Sungnam, Korea) and complementary DNA (cDNA) was subsequently synthesized utilizing the iScript ${ }^{\mathrm{TM}}$ cDNA Synthesis Kit (Biorad, Foster City, California, USA). mRNA levels of $\mathrm{CEBP} \alpha, \mathrm{CEBP} \beta$, sterol regulatory element binding protein-1c (SREBP1c), and PPAR were estimated by Real-time PCR in StepOnePlus equipment (Applied Biosystems, California, MA, USA) using 2X SYBR Green Master Mix (Takara, Shiga, Japan). Expression levels of mRNA were normalized using $\beta$-actin and analyzed to $\Delta \Delta \mathrm{Ct}$ methods for quantification. Primers for Primer sequences were designed as shown in Table 1.

\section{Statistical analysis}

All experiments were performed 4 times or 3 times, and the data is expressed as the mean \pm standard deviation (SD) for the independent experiments. The mean difference between the groups was analyzed by one-way ANOVA using GraphPad Prism 3.0 (Graphpad, San Diego, CA, USA). A $p$ value of less than 0.05 was considered statistically significant.

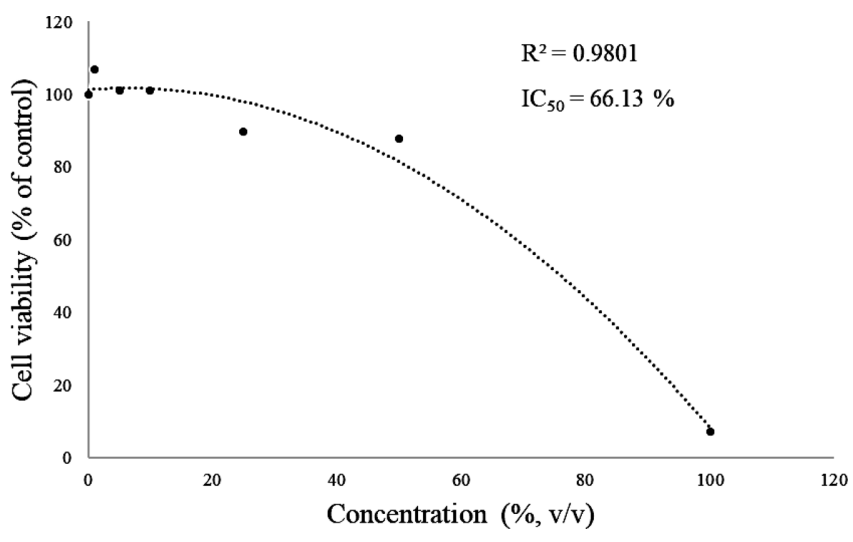

Fig. 1 The cell viability of LPM doses according to concentration and $\mathrm{IC}_{50}$ of LPM dose against in 3T3 adipocytes. The data results are offered as mean \pm SEM. $* * *$ means significantly different at $p<0.001$ from negative control

\section{Results and Discussion}

\section{Effect of LPM on cell viability in the 3T3-L1 adipocyte}

In order to measure the cell viability using an MTT assay, different concentrations of the LPM LB meso solution comprising of the Melissa officinalis extraction (LPM) were treated in the 3T3-L1 adipocytes for 4 hours. The cell viability of the 3T3 cell ranged from 106.9 to $87.8 \%$ of the control group with a 0 to $50 \%$ solution, but a $100 \%$ solution induced cell cytotoxicity by showing $7.1 \%$ of the control cell (Fig. 1). A concentration to inhibit $50 \%$ of the cell viability $\left(\mathrm{IC}_{50}\right)$ value was determined to be $66.13 \%$ (Fig. 1), which indicates that a $100 \%$ LPM solution could be specific with the decrease in the cell viability of the mature adipocytes. Further studies to investigate the effect of the LPM on the lipolysis and the lipid metabolism were further conducted within $50 \%$ of the LPM solution.

\section{Effect of LPM on the lipid accumulation in the 3T3-L1 adipocyte}

The inhibitory effect of the LPM on the lipid accumulation was confirmed by the red color with the oil red O staining in the 3T3L1 adipocytes (Fig. 2). It was observed that the addition of the LPM appeared to suppress the lipid accumulation compared to the negative controls in the representative images (Fig. 2A). According to this quantitative data obtained by quantifying the red colored portion, 10, 25, and 50\% LPM solution contained lipid contents of $91.75 \pm 2.34,86.85 \pm 0.89$, and $62.87 \pm 3.25 \%$, respectively, compared to the untreated control cells (Fig. 2B). It was significant that in a dose dependent manner it decreased by $1.2(p<0.01), 1.1(p<0.001)$, and 1.6-fold $(p<0.001)$ at 10,25 , and $50 \%$ of the solution, respectively. In contrast to the negative control group, the 
(A) Magnification $=200 \mathrm{X}$



(B)

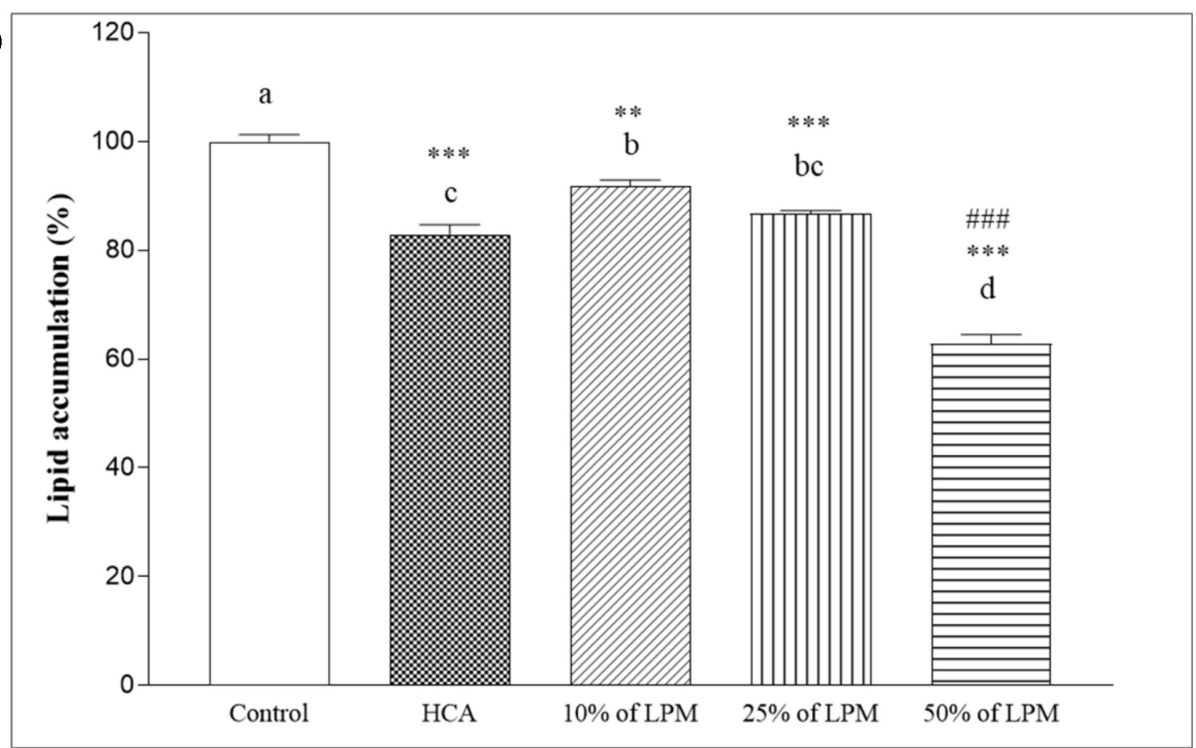

Fig. 2 The effects of the LPM on lipid accumulation in the 3T3-L1 adipocytes: Histology of the representative oil red O stained 3T3 cells sections (original magnification $\times 200$ ) (A) and the quantitative analysis of the lipid accumulation. The data results are offered as mean \pm SEM $(B)$. $* p<0.05$, ${ }^{* *} p<0.01$, and ${ }^{* * *} p<0.001$ versus the negative control group. ${ }^{\# \#} p<0.001$ versus the positive control. LPM; LPM LB meso solution containing Melissa officinalis $L$. leaf extract, HCA; hydroxycitric acid

treatment of the positive control group (hydroxycitric acid, HCA) had a decreased lipid content by $82.96 \%$. Particularly, a 50\% LPM solution was found to have a 1.3-fold decrease in lipid content compared to the HCA treated cells $(p<0.001)$. This implies that either the whole Melissa officinalis L., extracts or the specific components in the LPM could induce the inhibition of the lipid accumulation. Similar to our finding, it was revealed that Melissa officinalis $L$. extract induced a decrease in the lipid accumulation in the $3 \mathrm{~T} 3$ adipocytes [9].
Effect of LPM on the adipogenic gene expression in the 3T3L1 adipocyte

The mechanism of inhibiting the lipid accumulation by the LPM was further elucidated by the mRNA expression of the genes modulating adipogenic process $(\mathrm{CEBP} \alpha, \mathrm{CEBP} \beta, \mathrm{SREBP} 1 \mathrm{c}$, and PPAR $\gamma$ ). The LPM significantly induced the up-regulation of the gene expression for the CEBP $\beta(p<0.001)$ and the PPAR $\gamma$ $(p<0.05)$ compared to the control group (Figs. 3B, C). On the other hand, the treatment with the LPM decreased the mRNA 
(A)

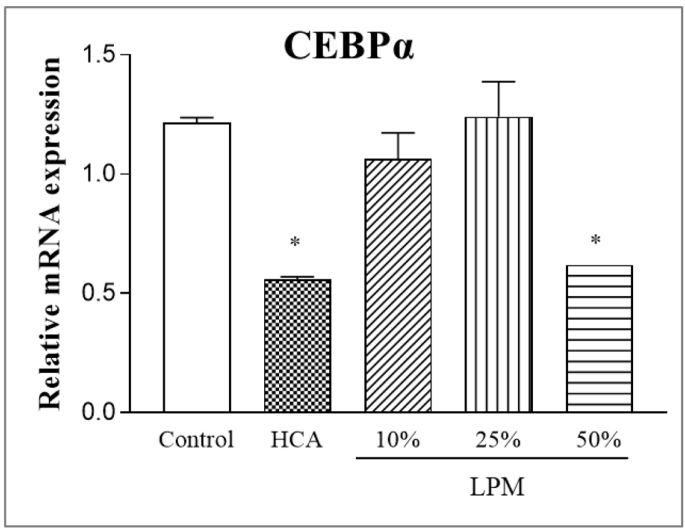

(C)

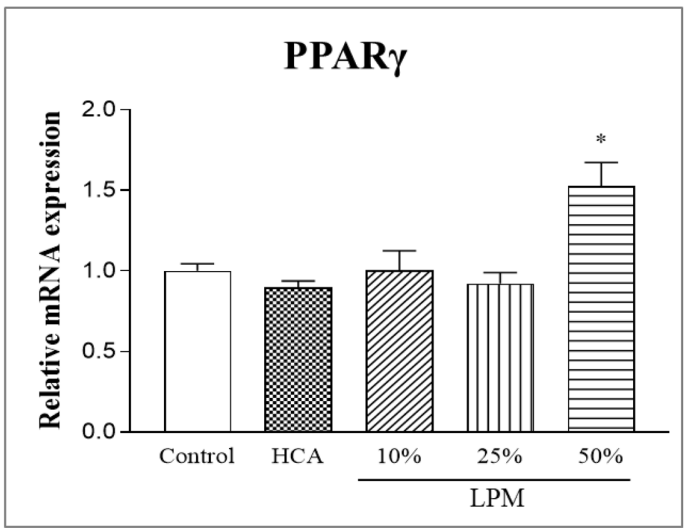

(B)

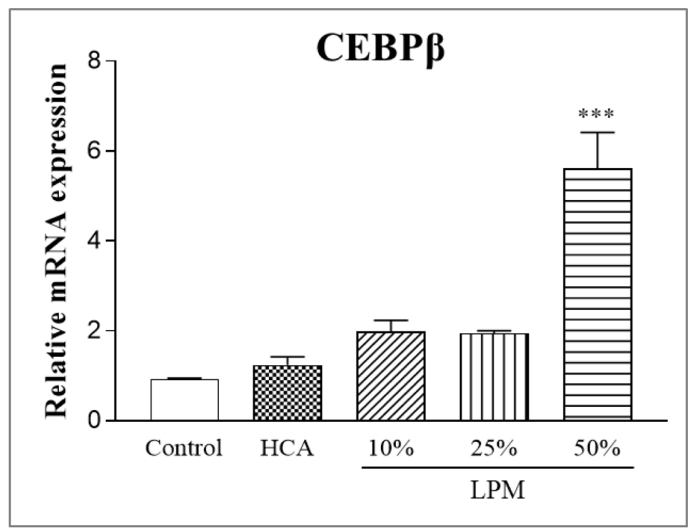

(D)



Fig. 3 The effect of the LPM on the gene expression of lipid metabolism-related transcription factors in 3T3-L1 adipocytes. The cell lysates were quantitation to real-time PCR for (A) CEBP $\alpha$, (B) CEBP $\beta$, (C) PPAR $\gamma$, and (D) SREBP1c. $\beta$-Actin was used as a loading control. The data results are offered as mean \pm SEM. ${ }^{*} p<0.05, * * p<0.01$, and $* * * p<0.001$ versus the negative control group. LPM; LPM LB meso solution containing Melissa officinalis $L$. leaf extract (LPM), HCA; hydroxycitric acid

expression of the genes CEBPá and SREBP1c (Figs. 3A, D). According to the gene expression data obtained, the positive control (HCA) significantly decreased for the CEBP $\alpha(p<0.05)$ and the SREBP1c $(p<0.01)$ compared to the negative control group. On the other hand, the expression of the CEBPâ and the PPAR $\gamma$ were not significantly affected and similar to the negative control group. The expression of the CEBP $\alpha$ was significantly decreased by 1.6 -fold with a 50\% LPM solution $(p<0.05)$, which was similar to the positive control group by 1.8 -fold. The expression of the SREBP1c was significantly decreased by 1.3 , 2.3, and 3.6 folds at a 10\% LPM solution ( $p<0.01)$, a 25\% LPM solution $(p<0.01)$, and a 50\% LPM solution $(p<0.001)$, respectively. Also, the SREBP1c 50\% LPM solution decreased by more than 2.5 folds than the treatment for the positive control group (HCA).

Among the CEBP, the CEBPâ was expressed early in the adipocyte differentiation program, and the activation of the CEBP $\beta$ promotes the CEBP $\alpha$ and the PPAR $\gamma$ expression [1]. Interestingly, our results found that the mRNA expression of the CEBP $\alpha$ was down-regulated, while the mRNA expression of the CEBP $\beta$ was upregulated by the LPM (Figs. 3A, B). It was also demonstrated that activation of the PPAR $\gamma$ expression seemed to be promoted according to the activating of the CEBP $\beta$. Previous studies also found increases in the hydrolysis of the triglyceriderich lipoproteins, secretion of the adiponectin, and insulin sensitivity in the mature adipocytes. White the adipose tissue (WAT) and the brown adipose tissue (BAT) have antagonistic functions and in contrast to the WAT, the BAT has the ability to release energy in the form of heat $[13,18]$. The appearance of brown-like cells at the WAT depot is caused by colds, exercise, or pharmacological treatment, such as the PPAR $\gamma$ agonists [13]. The previous study reported that activation of the PPAR $\gamma$ transcriptional function stimulates the brown adipogenesis, and the relatively large amounts of BAT were associated with weight loss [18]. The current study increased the expression of PPAR $\gamma$, which could be specific to the brown adipose tissue. The results from the current study concluded that $50 \%$ of the LPM solution seems to decrease the lipid accumulation by increasing the PPAR $\gamma$ expression and by also decreasing the CEBP $\alpha$ and the SREBP1 levels. The current study suggests the possible mechanisms of the LPM to inhibit adipogenesis, which include 1) adipogenic differentiation in the terminal stage could be inhibited by decreasing the CEBP $\alpha, 2)$ the BAT associated with weight loss could be induced by increasing 
the expression of the PPAR $\gamma$, and 3) the enzyme activity on the lipid synthesis could be suppressed by down-regulating the gene expression of the SREBP1c.

In conclusion, the current study investigated the effects of a hypodermic injectable solution compromising by Melissa officinalis $L$. leaf extract (LPM) on lipogenesis in the 3T3-L1 cells line. An LPM treatment that has up to a $50 \%$ solution reduced the lipid accumulation in the 3T3-L1 adipocytes. Regarding the adipogensis related transcription factors, the LPM up-regulated the expression of the PPAR $\gamma$ that promoting the hydrolysis of the triglyceriderich lipoproteins and the secretion of adiponectin and insulin sensitivity in the mature adipocytes. It also down-regulated the expression of the CEBP $\alpha$ and the SREBP1c that was responsible for the adipogenic differentiation in the terminal stage and lipid synthesis enzymes, respectively, which resulted in prevention of adipogenesis. The results from the current study suggest that the LPM could be useful ingredients for hypodermic injector ingredients to regulate obesity. Future studies on in vivo and clinical trials are needed for the safety and the efficacy to validate the results observed with in vitro models.

\section{References}

1. Banjare J, Raina P, Mansara P, Ghanekar RK, Bhalerao SJPm (2017) Triphala, regulates adipogenesis through modulation of expression of adipogenic genes in 3T3-L1 cell line. Pharmacogn Mag 13: S834

2. Muthukumaran P, Thiyagarajan G, Babu RA, Lakshmi BS (2018) Raffinose from Costus speciosus attenuates lipid synthesis through modulation of PPARs/SREBP1c and improves insulin sensitivity through PI3K/AKT. Chem-Biol Interact 284: 80-89

3. Kakkar AK, Dahiya N (2015) Drug treatment of obesity: current status and future prospects. Eur J Intern Med 26: 89-94

4. Tung YC, Hsieh PH, Pan MH, Ho CT (2017) Cellular models for the evaluation of the antiobesity effect of selected phytochemicals from food and herbs. J Food Drug Anal 25: 100-110

5. Marrelli M, Conforti F, Araniti F, Statti GA (2016) Effects of Saponins on Lipid Metabolism: A Review of Potential Health Benefits in the Treatment of Obesity. Molecules 21: 1404
6. Jemai R, Drira R, Fetoui H, Makni M, Sakamoto KJF, Sciences N (2018) Colocynth (Citrullus colocynthis) Flesh Extract Suppresses Adipogenesis by Down-Regulating Adipogenic Transcription Factors and Their Target Genes in 3T3-L1 Preadipocytes. 9: 1014

7. de Carvalho NC, Correa-Angeloni MJ, Leffa DD, Moreira J, Nicolau V, de Aguiar Amaral P, Rossatto AE, de Andrade VM (2011) Evaluation of the genotoxic and antigenotoxic potential of Melissa officinalis in mice. Genet Mol Biol 34: 290-297

8. Shakeri A, Sahebkar A, Javadi BJJoe (2016) Melissa officinalis L.-A review of its traditional uses, phytochemistry and pharmacology. J Ethnopharmacol 188: 204-228

9. Woo S, Yoon M, Kim J, Hong Y, Kim MY, Shin SS, Yoon M (2016) The anti-angiogenic herbal extract from Melissa officinalis inhibits adipogenesis in 3T3-L1 adipocytes and suppresses adipocyte hypertrophy in high fat diet-induced obese C57BL/6J mice. J Ethnopharmacol 178: 238-250

10. Faulhaber J, Sandhofer M, Weiss C, Sattler G, Sadick NS (2019) Effective noninvasive body contouring by using a combination of cryolipolysis, injection lipolysis, and shock waves. J Cosmet DermatolUs 18: 1014-1019

11. Yoo SR, Lee MY, Kang BK, Shin HK, Jeong SJ (2016) Soshiho-Tang Aqueous Extract Exerts Antiobesity Effects in High Fat Diet-Fed Mice and Inhibits Adipogenesis in 3T3-L1 Adipocytes. Evid Based Complement Alternat Med 2016: 2628901

12. Kucharski M, Kaczor U (2018) PPAR alpha and PPAR gamma as main regulators of fatty acid metabolism. Postep Hig Med Dosw 72: 853-860

13. Lanzi CR, Perdicaro DJ, Landa MS, Fontana A, Antonioni A, Miatello RM, Oteiza PI, Prieto MAV (2018) Grape pomace extract induced beige cells in white adipose tissue from rats and in 3T3-L1 adipocytes. J Nutr Biochem 56: 224-233

14. Marion-Letellier R, Savoye G, Ghosh S (2016) Fatty acids, eicosanoids and PPAR gamma. Eur J Pharmacol 785: 44-49

15. Echeverria F, Ortiz M, Valenzuela R, Videla LA (2016) Long-chain polyunsaturated fatty acids regulation of PPARs, signaling: Relationship to tissue development and aging. Prostag Leukotr Ess 114: 28-34

16. Lefils-Lacourtablaise J, Socorro M, Géloën A, Daira P, Debard C, Loizon E, Guichardant M, Dominguez Z, Vidal H, Lagarde MJPo (2013) The eicosapentaenoic acid metabolite 15 -deoxy- $\delta 12$, 14-prostaglandin J3 increases adiponectin secretion by adipocytes partly via a PPAR $\gamma$ dependent mechanism. PLoS One 8

17. Shimano H, Sato RJNRE (2017) SREBP-regulated lipid metabolism: convergent physiology-divergent pathophysiology. Nat. Rev. Endocrinol 13: 710

18. Saely CH, Geiger K, Drexel H (2012) Brown versus white adipose tissue: a mini-review. Gerontology 58: 15-23 